Published in final edited form as:

Nat Genet. ; 43(10): 1005-1011. doi:10.1038/ng.922.

\title{
Genome-wide association study identifies six new loci influencing pulse pressure and mean arterial pressure
}

Louise V Wain ${ }^{1,2,180}$, Germaine C Verwoert ${ }^{3,4,180}$, Paul F O'Reilly ${ }^{5,180}$, Gang Shi6,7,180, Toby Johnson $^{8,180}$, Andrew D Johnson ${ }^{9,10}$, Murielle Bochud ${ }^{11,12}$, Kenneth M Rice ${ }^{13}$, Peter Henneman ${ }^{14}$, Albert V Smith ${ }^{15,16}$, Georg B Ehret ${ }^{17,18,19}$, Najaf Amin ${ }^{20}$, Martin G Larson ${ }^{9,21}$, Vincent Mooser ${ }^{22}$, David Hadley ${ }^{23,24}$, Marcus Dörr ${ }^{25}$, Joshua C Bis ${ }^{26}$, Thor Aspelund 15,16, Tõnu Esko ${ }^{27,28,29}$, A Cecile JW Janssens ${ }^{20}$, Jing Hua Zhao ${ }^{30}$, Simon Heath ${ }^{31}$, Maris Laan ${ }^{29}$, Jingyuan Fu ${ }^{32,33}$, Giorgio Pistis ${ }^{34}$, Jian'an Luan ${ }^{30}$, Pankaj Arora ${ }^{35}$, Gavin Lucas ${ }^{36}$, Nicola Pirastu $^{37}$, Irene Pichler ${ }^{38}$, Anne U Jackson ${ }^{39}$, Rebecca J Webster ${ }^{40}$, Feng Zhang ${ }^{41}$, John F Peden $^{42,43}$, Helena Schmidt ${ }^{44}$, Toshiko Tanaka ${ }^{45}$, Harry Campbell ${ }^{46}$, Wilmar Igl $\left.\right|^{47}$, Yuri Milaneschi ${ }^{45}$, Jouke-Jan Hotteng ${ }^{48}$, Veronique Vitart ${ }^{49}$, Daniel I Chasman ${ }^{50,51}$, Stella Trompet $^{52,53}$, Jennifer L Bragg-Gresham ${ }^{39}$, Behrooz Z Alizadeh ${ }^{32}$, John C Chambers ${ }^{5,54}$, Xiuqing Guo ${ }^{55}$, Terho Lehtimäki ${ }^{56}$, Brigitte Kühnel ${ }^{57}$, Lorna M Lopez ${ }^{58,59}$, Ozren Polašek ${ }^{60}$, Mladen Boban ${ }^{61}$, Christopher P Nelson ${ }^{62}$, Alanna C Morrison ${ }^{63}$, Vasyl Pihur ${ }^{17}$, Santhi K Ganesh $^{64}$, Albert Hofman ${ }^{20}$, Suman Kundu ${ }^{20}$, Francesco US Mattace-Raso ${ }^{20,3}$, Fernando Rivadeneira $^{3,4}$, Eric JG Sijbrands ${ }^{20,3}$, Andre G Uitterlinden ${ }^{3,4}$, Shih-Jen Hwang $9,65,10$, Ramachandran S Vasan ${ }^{9,66}$, Thomas J Wang ${ }^{9,67}$, Sven Bergmann ${ }^{68,69}$, Peter Vollenweider $^{70}$, Gérard Waeber ${ }^{70}$, Jaana Laitinen ${ }^{71}$, Anneli Pouta ${ }^{72}$, Paavo Zitting ${ }^{73}$, Wendy L McArdle $^{74}$, Heyo K Kroemer ${ }^{75}$, Uwe Völker ${ }^{76}$, Henry Völzke ${ }^{77}$, Nicole L Glazer ${ }^{78}$, Kent D Taylor $^{55}$, Tamara B Harris ${ }^{79}$, Helene Alavere ${ }^{27}$, Toomas Haller $^{27}$, Aime Keis ${ }^{27}$, Mari-Liis Tammesoo 27 , Yurii Aulchenko 20 , Inês Barroso 80,81 , Kay-Tee Khaw ${ }^{82}$, Pilar Galan $83,84,85$, Serge Hercberg ${ }^{83,84,85}$, Mark Lathrop ${ }^{31}$, Susana Eyheramendy ${ }^{86}$, Elin Org $^{29}$, Siim Sõber ${ }^{29}$, Xiaowen Lu ${ }^{32}$, Ilja M Nolte ${ }^{32}$, Brenda W Penninx ${ }^{87,88,89}$, Tanguy Corre ${ }^{34}$, Corrado Masciullo $^{34}$, Cinzia Sala ${ }^{34}$, Leif Groop ${ }^{90}$, Benjamin F Voight ${ }^{91}$, Olle Melander ${ }^{92}$, Christopher J O'Donnell ${ }^{93}$, Veikko Salomaa ${ }^{94}$, Adamo Pio d'Adamo ${ }^{37}$, Antonella Fabretto ${ }^{95}$, Flavio Faletra $^{95}$, Sheila Ulivi ${ }^{37}$, M Fabiola Del Greco ${ }^{38}$, Maurizio Facheris ${ }^{38}$, Francis S Collins ${ }^{96}$, Richard N Bergman ${ }^{97}$, John P Beilby $98,99,100$, Joseph Hung ${ }^{101,100}$, A William Musk $^{100,102,103}$, Massimo Mangino ${ }^{41}$, So-Youn Shin ${ }^{80,41}$, Nicole Soranzo ${ }^{80,41}$, Hugh Watkins $^{42,43}$, Anuj Goel ${ }^{42,43}$, Anders Hamsten ${ }^{104}$, Pierre Gider ${ }^{44}$, Marisa Loitfelder ${ }^{105}$, Marion Zeginigg ${ }^{44}$, Dena Hernandez ${ }^{106}$, Samer S Najjar ${ }^{107,108}$, Pau Navarro ${ }^{49}$, Sarah H Wild $^{46}$, Anna Maria Corsi ${ }^{109}$, Andrew Singleton ${ }^{106}$, Eco JC de Geus ${ }^{110}$, Gonneke Willemsen ${ }^{110}$, Alex N Parker ${ }^{111}$, Lynda M Rose ${ }^{50}$, Brendan Buckley ${ }^{112}$, David Stott ${ }^{113}$, Marco Orru ${ }^{114}$, Manuela Uda ${ }^{114}$, LifeLines Cohort Study, Melanie M van der Klauw ${ }^{115}$, Weihua Zhang ${ }^{5,54}$, Xinzhong Li ${ }^{5}$, James Scott ${ }^{116}$, Yii-Der Ida Chen ${ }^{55}$, Gregory L Burke ${ }^{117}$, Mika Kähönen $^{118}$, Jorma Viikari ${ }^{119}$, Angela Döring ${ }^{120,121}$, Thomas Meitinger ${ }^{122,123}$, Gail Davies $^{59}$, John M Starr ${ }^{58,124}$, Valur Emilsson ${ }^{15}$, Andrew Plump ${ }^{125}$, Jan H Lindeman ${ }^{126}$, Peter AC 't Hoen ${ }^{127,128}$, Inke R König ${ }^{129}$, EchoGen consortium ${ }^{179}$, Janine F Felix ${ }^{20,4,130}$, Robert Clarke $^{131}$, Jemma C Hopewell ${ }^{131}$, Halit Ongen ${ }^{42}$, Monique Breteler ${ }^{20}$, Stéphanie

Correspondence to: Martin D Tobin; Paul Elliott; Cornelia M van Duijn.

${ }^{179} \mathrm{~A}$ list of consortium members is supplied in the Supplementary Note.

${ }^{180}$ These authors contributed equally.

Competing Financial Interests

A.C. is managed by Johns Hopkins Medicine. I.B. and spouse own stock in Incyte Ltd and GlaxoSmithKline. A.N.P is an employee of Amgen. G.F.M. is owner of Cardiovascular Engineering, Inc, a company that designs and manufactures devices that measure vascular stiffness. The company uses these devices in clinical studies that evaluate the effects of diseases and interventions on vascular stiffness. V.M. is an employee of GlaxoSmithKline plc. A.Plump is an employee of Merck and Co, Inc. 
Debette $^{132}$, Anita L DeStefano ${ }^{133}$, Myriam Fornage ${ }^{134}$, AortaGen Consortium ${ }^{179}$, Gary F Mitchell ${ }^{135}$, CHARGE Consortium Heart Failure Working Group ${ }^{179}$, Nicholas L Smith $^{136,137,138}$, KidneyGen consortium ${ }^{179}$, Hilma Holm ${ }^{139}$, Kari Stefansson ${ }^{139,140}$, Gudmar Thorleifsson ${ }^{139}$, Unnur Thorsteinsdottir ${ }^{139,140}$, CKDGen consortium ${ }^{179}$, Cardiogenics consortium ${ }^{179}$, CardioGram ${ }^{179}$, Nilesh J Samani62,141, Michael Preuss ${ }^{142,129}$, Igor Rudan $^{46,143}$, Caroline Hayward ${ }^{49}$, lan J Deary ${ }^{58,59}$, H-Erich Wichmann ${ }^{120,144}$, Olli T Raitakari ${ }^{145}$, Walter Palmas ${ }^{146}$, Jaspal S Kooner ${ }^{116,54}$, Ronald P Stolk ${ }^{147}$, J Wouter Jukema $^{52,148,149}$, Alan F Wright ${ }^{49}$, Dorret I Boomsma ${ }^{110}$, Stefania Bandinelli ${ }^{150}$, Ulf B Gyllensten $^{47}$, James F Wilson ${ }^{46}$, Luigi Ferrucci ${ }^{45}$, Reinhold Schmidt ${ }^{105}$, Martin Farrall ${ }^{42,43}$, Tim D Spector 41 , Lyle J Palmer ${ }^{40,100,151,152}$, Jaakko Tuomilehto ${ }^{153,154,155}$, Arne Pfeufer ${ }^{38,156,157}$, Paolo Gasparini 95,37 , David Siscovick ${ }^{158,136,26}$, David Altshuler ${ }^{159,160,91,161}$, Ruth JF Loos ${ }^{30}$, Daniela Toniolo ${ }^{34,162}$, Harold Snieder ${ }^{32}$, Christian Gieger $^{57}$, Pierre Meneton ${ }^{163}$, Nicholas J Wareham ${ }^{30}$, Ben A Oostra ${ }^{164}$, Andres Metspalu27,28,29, Lenore Launer ${ }^{165}$, Rainer Rettig ${ }^{166}$, David P Strachan ${ }^{23}$, Jacques S Beckmann ${ }^{68,167}$, Jacqueline CM Witteman ${ }^{20,4}$, Jeanette Erdmann ${ }^{142}$, Ko Willems van

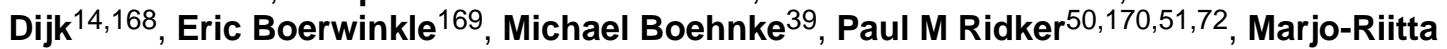
Jarvelin ${ }^{171,172,173}$, Aravinda Chakravarti ${ }^{17}$, Goncalo R Abecasis ${ }^{39}$, Vilmundur Gudnason ${ }^{15,16}$, Christopher Newton-Cheh ${ }^{35,91}$, Daniel Levy $9,65,10$, Patricia B Munroe ${ }^{8,180}$, Bruce M Psaty26,136,174,138,180, Mark J Caulfield ${ }^{8,180}$, Dabeeru C Rao6,7,175,176,180, Martin D Tobin $^{1,2,180}$, Paul Elliott ${ }^{177,5,180}$, and Cornelia M van Duijn $20,178,4,180$

${ }^{1}$ Department of Health Sciences, University of Leicester, Leicester, UK ${ }^{2}$ Department of Genetics, University of Leicester, Leicester, UK ${ }^{3}$ Department of Internal Medicine, Erasmus University Medical Center, Rotterdam, The Netherlands ${ }^{4}$ Netherlands Genomics Initiative (NGI)-sponsored Netherlands Consortium for Healthy Aging (NCHA) ${ }^{5}$ Department of Epidemiology and Biostatistics, School of Public Health, Imperial College London, London, UK ${ }^{6}$ Division of Biostatistics, Washington University in St. Louis, School of Medicine, Saint Louis, Missouri, USA ${ }^{7}$ Department of Genetics, Washington University in St. Louis, School of Medicine, Saint Louis, Missouri, USA ${ }^{8}$ Clinical Pharmacology and The Genome Centre, William Harvey Research Institute, Barts and The London School of Medicine and Dentistry, Queen Mary University of London, London, UK ${ }^{9}$ Framingham Heart Study, Framingham, Massachusetts, USA ${ }^{10}$ National Heart, Lung and Blood Institute, Bethesda, Maryland, USA ${ }^{11}$ Institute of Social and Preventive Medicine (IUMSP), Centre Hospitalier Universitaire Vaudois, 1005 Lausanne, Switzerland ${ }^{12}$ University of Lausanne, Lausanne, Switzerland ${ }^{13}$ Department of Biostatistics, University of Washington, Seattle, Washington, USA ${ }^{14}$ Department of Human Genetics, Leiden University Medical Center, Leiden, the Netherlands ${ }^{15}$ Icelandic Heart Association, Kopavogur, Iceland ${ }^{16}$ University of Iceland, Reykajvik, Iceland ${ }^{17}$ Center for Complex Disease Genomics, McKusickNathans Institute of Genetic Medicine, Johns Hopkins University School of Medicine, Baltimore, Maryland, USA ${ }^{18}$ Institute of Social and Preventive Medicine, Lausanne University Hospital, Lausanne, Switzerland ${ }^{19}$ Cardiology, Department of Medicine, Geneva University Hospital, Geneva, Switzerland ${ }^{20}$ Department of Epidemiology, Erasmus University Medical Center, Rotterdam, The Netherlands ${ }^{21}$ Department of Mathematics, Boston University, Boston, Massachusetts, USA ${ }^{22}$ Genetics Division R\&D, GlaxoSmithKline, King of Prussia, Pennsylvania, USA ${ }^{23}$ Division of Community Health Sciences, St George's, University of London, London, UK ${ }^{24}$ Pediatric Epidemiology Center, University of South Florida, Tampa, Florida, USA ${ }^{25}$ Department of Internal Medicine B, Ernst-Moritz-Arndt-University Greifswald, Greifswald, Germany ${ }^{26}$ Cardiovascular Health Research Unit, Division of Internal Medicine, Department of Medicine, University of Washington, Seattle, Washington, USA ${ }^{27}$ Estonian Genome Center, University of Tartu, Tartu, Estonia ${ }^{28}$ Estonian Biocenter, Tartu, Estonia ${ }^{29}$ Institute of Molecular and Cell Biology, University of Tartu, Tartu, Estonia ${ }^{30} \mathrm{MRC}$ Epidemiology Unit, Institute of Metabolic Science, Cambridge, UK ${ }^{31}$ Centre National de Génotypage, Commissariat à L'Energie Atomique, Institut de Génomique, Evry, France ${ }^{32}$ Unit of Genetic Epidemiology and Bioinformatics, 
Department of Epidemiology, University Medical Center Groningen, University of Groningen, Groningen, The Netherlands ${ }^{33}$ Department of Genetics, University Medical Center Groningen, University of Groningen, Groningen, The Netherlands ${ }^{34}$ Division of Genetics and Cell Biology, San Raffaele Scientific Institute, Milano, Italy ${ }^{35}$ Center for Human Genetic Research, Cardiovascular Research Center, Massachusetts General Hospital, Boston, Massachusetts, USA ${ }^{36}$ Cardiovascular Epidemiology and Genetics, Institut Municipal d'Investigacio Medica, Barcelona Biomedical Research Park, Barcelona, Spain ${ }^{37}$ Medical Genetics IRCCS Burlo Garofolo/ Università degli Studi di Trieste, Trieste, Italy ${ }^{38}$ Institute of Genetic Medicine, European Academy Bozen/Bolzano (EURAC), Bolzano, Italy. Affiliated Institute of the University of Lübeck, Lübeck, Germany ${ }^{39}$ Department of Biostatistics, Center for Statistical Genetics, University of Michigan, Ann Arbor, Michigan, USA ${ }^{40}$ Centre for Genetic Epidemiology and Biostatistics, University of Western Australia, Crawley, Western Australia, Australia ${ }^{41}$ Department of Twin Research and Genetic Epidemiology, King's College London, London, UK ${ }^{42}$ Department of Cardiovascular Medicine, The Wellcome Trust Centre for Human Genetics, University of Oxford, Oxford, UK ${ }^{43}$ Department of Cardiovascular Medicine, University of Oxford, John Radcliffe Hospital, Headington, Oxford, UK ${ }^{44}$ Institute of Molecular Biology and Biochemistry, Medical University Graz, Graz, Austria ${ }^{45} \mathrm{Clinical}$ Research Branch, National Institute on Aging, Baltimore, Maryland, USA ${ }^{46}$ Centre for Population Health Sciences, University of Edinburgh, Edinburgh, UK ${ }^{47}$ Department of Genetics and Pathology, Rudbeck Laboratory, Uppsala University, Uppsala, Sweden ${ }^{48} \mathrm{NCA}$ institute, Department of Biological Psychology, VU University, Amsterdam, The Netherlands ${ }^{49} \mathrm{MRC}$ Human Genetics Unit, Institute of Genetics and Molecular Medicine, Western General Hospital, Edinburgh, UK ${ }^{50}$ Division of Preventive Medicine, Brigham and Women's Hospital, Boston, Massachusetts, USA ${ }^{51}$ Harvard Medical School, Boston, Massachusetts, USA ${ }^{52}$ Department of Cardiology, Leiden University Medical Center, Leiden, The Netherlands ${ }^{53}$ Department of Gerontology and Geriatrics, Leiden University Medical Center, Leiden, The Netherlands ${ }^{54}$ Ealing Hospital NHS Trust, Middlesex, UK ${ }^{55}$ Medical Genetics Institute, CedarsSinai Medical Center, Los Angeles, California, USA ${ }^{56}$ Department of Clinical Chemistry, University of Tampere and Tampere University Hospital, Tampere, Finland ${ }^{57}$ Institute of Genetic Epidemiology, Helmholtz Zentrum München - German Research Center for Environmental Health, Neuherberg, Germany ${ }^{58}$ Centre for Cognitive Ageing and Cognitive Epidemiology, The University of Edinburgh, Edinburgh, UK ${ }^{59}$ Department of Psychology, The University of Edinburgh, Edinburgh, UK ${ }^{60}$ Department of Public Health, Medical School, University of Split, Split, Croatia ${ }^{61}$ Department of Pharmacology, Medical School, University of Split, Split, Croatia ${ }^{62}$ Department of Cardiovascular Sciences, University of Leicester, Glenfield Hospital, Leicester, UK ${ }^{63}$ Division of Epidemiology, Human Genetics and Environmental Sciences, School of Public Health, University of Texas at Houston Health Science Center, Houston, Texas, USA ${ }^{64}$ Department of Internal Medicine, Division of Cardiovascular Medicine, University of Michigan Medical Center, Ann Arbor, Michigan, USA ${ }^{65}$ Center for Population Studies, National Heart Lung, and Blood Institute, Bethesda, Maryland, USA ${ }^{66}$ Division of Epidemiology and Prevention, Boston University School of Medicine, Boston, Massachusetts, USA ${ }^{67}$ Division of Cardiology, Massachusetts General Hospital, Boston, Massachusetts, USA ${ }^{68}$ Département de Génétique Médicale, Université de Lausanne, Lausanne, Switzerland ${ }^{69}$ Swiss Institute of Bioinformatics, Lausanne, Switzerland ${ }^{70}$ Department of Internal Medicine, Centre Hospitalier Universitaire Vaudois, Lausanne, Switzerland ${ }^{71}$ Finnish Institute of Occupational Health, Oulu, Finland ${ }^{72}$ National Institute for Health and Welfare, Oulu, Finland ${ }^{73}$ Department of Physiatrics, Lapland Central Hospital, Rovaniemi, Finland ${ }^{74}$ ALSPAC Laboratory, Department of Social Medicine, University of Bristol, Bristol, UK ${ }^{75}$ Institute of Pharmacology, Ernst-Moritz-Arndt-University Greifswald, Greifswald, Germany ${ }^{76}$ Institute for Genetics and Functional Genomics, Ernst-MoritzArndt-University Greifswald, Greifswald, Germany ${ }^{77}$ Institute for Community Medicine, ErnstMoritz-Arndt-University Greifswald, Greifswald, Germany ${ }^{78}$ Section of Preventive Medicine and Epidemiology, Department of Medicine, Boston University School of Medicine, Boston, 
Massachusetts, USA ${ }^{79}$ National Institute of Aging's Laboratory for Epidemiology, Demography and Biometry, Bethesda, Maryland, USA ${ }^{80}$ Wellcome Trust Sanger Institute, Wellcome Trust Genome Campus, Hinxton, Cambridge, UK ${ }^{81}$ University of Cambridge Metabolic Research Labs, Institute of Metabolic Science, Addenbrooke's Hospital, Cambridge, UK ${ }^{82}$ Department of Public Health and Primary Care, Institute of Public Health, University of Cambridge, Cambridge, UK ${ }^{83}$ U557 Institut National de la Santé et de la Recherche Médicale, Paris, France ${ }^{84}$ U1125 Institut National de la Recherche Agronomique, Paris, France ${ }^{85}$ Université Paris 13, Bobigny, France ${ }^{86}$ Department of Statistics, Pontificia Universidad Catolica de Chile, Santiago, Chile ${ }^{87}$ Department of Psychiatry/EMGO Institute/Neuroscience Campus, VU University Medical Centre, Amsterdam, The Netherlands ${ }^{8}$ Department of Psychiatry, Leiden University Medical Centre, Leiden, The Netherlands ${ }^{89}$ Department of Psychiatry, University Medical Center Groningen, University of Groningen, Groningen, The Netherlands ${ }^{90}$ Department of Clinical Sciences, Diabetes and Endocrinology Research Unit, Lund University, University Hospital Malmö, Malmö, Sweden ${ }^{91}$ Program in Medical and Population Genetics, Broad Institute of Harvard and MIT, Cambridge, Massachusetts, USA ${ }^{92}$ Department of Clinical Sciences, Hypertension and Cardiovascular Diseases, University Hospital, Malmö, Lund University, Malmö 20502, Sweden ${ }^{93}$ National Heart, Lung and Blood Institute and its Framingham Heart Study, 73 Mount Wayte Ave., Suite

${ }^{94}$ Department of Chronic Disease Prevention, THL-National Institute for Health and Welfare, Helsinki, Finland ${ }^{95}$ IRCSS Burlo Garofolo Medical Genetics, Trieste, Italy ${ }^{96}$ National Human Genome Research Institute, National Institutes of Health, Bethesda, Maryland, USA ${ }^{97}$ Department of Physiology and Biophysics, Keck School of Medicine, University of Southern California, Los Angeles, California, USA ${ }^{98}$ Pathology and Laboratory Medicine, University of Western Australia, Crawley, Western Australia, Australia ${ }^{99}$ Molecular Genetics, PathWest Laboratory Medicine, Nedlands, Western Australia, Australia ${ }^{100}$ Busselton Population Medical Research Foundation, Sir Charles Gairdner Hospital, Nedlands, Western Australia, Australia ${ }^{101}$ School of Medicine and Pharmacology, University of Western Australia, Crawley, Western Australia, Australia ${ }^{102}$ School of Medicine and Pharmcology, University of Western Australia, Crawley, Western Australia, Australia ${ }^{103}$ Department of Respiratory Medicine, Sir Charles Gairdner Hospital, Nedlands, Western Australia, Australia ${ }^{104}$ Atherosclerosis Research Unit, Department of Medicine, Karolinska Institutet, Karolinska University Hospital, Stockholm, Sweden ${ }^{105}$ Department of Neurology Section of Special Neurology, Medical University Graz, Graz, Austria ${ }^{106}$ National Institute of Aging's Laboratory of Neurogenetics, Bethesda, Maryland, USA

${ }^{107}$ Laboratory of Cardiovascular Science, Intramural Research Program, National Institute on Aging, $\mathrm{NIH}$, Baltimore, Maryland, USA ${ }^{108} \mathrm{Cardiovascular}$ Research Institute, MedStar Health Research Institute, Washington Hospital Center, Washington DC, USA ${ }^{109}$ Tuscany Regional Health Agency, Florence, Italy ${ }^{110}$ EMGO+ institute, Department of Biological Psychology, VU University, Amsterdam, The Netherlands ${ }^{111}$ Amgen, Cambridge, Massachusetts, USA ${ }^{112}$ Department of Pharmacology and Therapeutics, University College Cork, Cork, Ireland ${ }^{113}$ Institute of Cardiovascular and Medical Sciences, School of Medicine, University of Glasgow, UK ${ }^{114}$ Istituto di Neurogenetica e Neurofarmacologia, Consiglio Nazionale delle Ricerche, Monserrato, Italy ${ }^{115}$ Department of Endocrinology, University Medical Center Groningen, University of Groningen, The Netherlands ${ }^{116}$ National Heart and Lung Institute, Imperial College London, Hammersmith Hospital, London, UK ${ }^{117}$ Division of Public Health Sciences, Wake Forest University School of Medicine, Winston-Salem, North Carolina, USA ${ }^{118}$ Department of Clinical Physiology, University of Tampere and Tampere University Hospital, Tampere, Finland ${ }^{119}$ Department of Medicine, University of Turku and Turku University Hospital, Turku, Finland ${ }^{120}$ Institute of Epidemiology I, Helmholtz Zentrum München - German Research Center for Environmental Health, Neuherberg, Germany ${ }^{121}$ Institute of Epidemiology II, Helmholtz Zentrum München - German Research Center for Environmental Health, Neuherberg, Germany ${ }^{122}$ Institute of Human Genetics, Helmholtz Zentrum München, German Research Centre for Environmental Health, Neuherberg, Germany ${ }^{123}$ Institute of Human Genetics, Technische Universität München, 
Munich, Germany ${ }^{124}$ Geriatric Medicine Unit, The University of Edinburgh, Royal Victoria, Edinburgh, UK ${ }^{125}$ Cardiovascular Disease Franchise, Merck Research Laboratory, Rahway, New Jersey, USA ${ }^{126}$ Department of Vascular Surgery, Leiden University Medical Center, Leiden, The Netherlands ${ }^{127}$ Department of Human Genetics, Leiden University Medical Center, Leiden, The Netherlands ${ }^{128}$ Leiden Genome Technology Center, Leiden University Medical Center, Leiden, The Netherlands ${ }^{129}$ Institut für Medizinische Biometrie und Statistik, Universität zu Lübeck, Lübeck, Germany ${ }^{130}$ German Cancer Research Center, Division of Clinical Epidemiology and Aging Research, Heidelberg, Germany ${ }^{131}$ Clinical Trial Service Unit and Epidemiological Studies Unit, University of Oxford, Oxford, UK ${ }^{132}$ Department of Neurology, Boston University School of Medicine, Boston, Massachusetts, USA ${ }^{133}$ Boston University School of Public Health, Boston, Massachusetts, USA ${ }^{134}$ Brown Foundation Institute of Molecular Medicine and Human Genetics Center, University of Texas Health Science Center at Houston, Houston, Texas, USA ${ }^{135}$ Cardiovascular Engineering, Inc., Norwood, Massachusetts, USA ${ }^{136}$ Department of Epidemiology, University of Washington, Seattle, Washington, USA ${ }^{137}$ Seattle Epidemiologic Research and Information, Center of the Department of Veterans Affairs Office of Research and Development, Seattle, Washington, USA ${ }^{138}$ Group Health Research Institute, Group Health Cooperative, Seattle, Washington, USA ${ }^{139}$ deCODE genetics Inc, Reykjavik, Iceland ${ }^{140}$ Faculty of Medicine, University of Iceland, Reykjavik, Iceland ${ }^{141}$ Leicester NIHR Biomedical Research Unit in Cardiovascular Disease, Glenfield Hospital, Leicester, UK ${ }^{142}$ Medizinische Klinik II, Universität zu Lübeck, Lübeck, Germany ${ }^{143}$ Croatian Centre for Global Health, University of Split Medical School, Split, Croatia ${ }^{144}$ Institute of Medical Informatics, Biometry and Epidemiology, LudwigMaximilians-Universität and Klinikum Grosshadern, Munich, Germany ${ }^{145}$ Research Centre of Applied and Preventive Cardiovascular Medicine, University of Turku and the Department of Clinical Physiology, Turku University Hospital, Turku, Finland ${ }^{146}$ Columbia University, New York, New York, USA ${ }^{147}$ Department of Epidemiology, University Medical Center Groningen, University of Groningen, The Netherlands ${ }^{148}$ Durrer Center for Cardiogenetic Research, Amsterdam, The Netherlands ${ }^{149}$ Interuniversity Cardiology Institute of the Netherlands, Utrecht, the Netherlands ${ }^{150}$ Geriatric Unit, Azienda Sanitaria Firenze (ASF), Florence, Italy ${ }^{151}$ Ontario Institute for Cancer Research, Toronto, Canada ${ }^{152}$ Samuel Lunenfeld Research Institute, Toronto, Canada ${ }^{153}$ National Institute for Health and Welfare, Diabetes Prevention Unit, Helsinki, Finland ${ }^{154} \mathrm{Hjelt}$ Institute, Department of Public Health, University of Helsinki, 00014 Helsinki, Finland ${ }^{155}$ South Ostrobothnia Central Hospital, Seinajoki, Finland ${ }^{156}$ Institute of Human Genetics, Klinikum rechts der Isar der Technischen Universität München, Munich, Germany ${ }^{157}$ Institute of Human Genetics, Helmholtz Zentrum München, Neuherberg, Germany ${ }^{158}$ Department of Medicine, University of Washington, Seattle, Washington, USA ${ }^{159}$ Department of Medicine, Harvard Medical School, Boston, Massachusetts, USA ${ }^{160}$ Department of Genetics, Harvard Medical School, Boston, Massachusetts, USA ${ }^{161}$ Diabetes Unit, Massachusetts General Hospital, Boston, Massachusetts, USA ${ }^{162}$ Institute of Molecuar Genetics_CNR, Pavia, Italy ${ }^{163}$ U872 Institut National de la Santé et de la Recherche Médicale, Centre de Recherche des Cordeliers, Paris, France ${ }^{164}$ Department of Clinical Genetics, Erasmus University Medical Center, Rotterdam, The Netherlands ${ }^{165}$ National Institute of Aging's Laboratory for Epidemiology, Demography and Biometry, Bethesda, Marylands, USA ${ }^{166}$ Institute of Physiology, Ernst-Moritz-Arndt-University Greifswald, Greifswald, Germany ${ }^{167}$ Service of Medical Genetics, Centre Hospitalier Universitaire Vaudois, Lausanne, Switzerland ${ }^{168}$ Department of Internal Medicine, Leiden University Medical Center, Leiden, the Netherlands ${ }^{169}$ Human Genetics Center, Houston, Texas, USA ${ }^{170}$ Division of Cardiology, Brigham and Women's Hospital, Boston, Massachusetts, USA ${ }^{171}$ Department of Biostatistics and Epidemiology, School of Public Health, Imperial College London, Norfolk Place, London, UK ${ }^{172}$ Institute of Health Sciences, University of Oulu, University of Oulu, Finland ${ }^{173}$ Biocenter, University of Oulu, Oulu, Finland ${ }^{174}$ Department of Health Services, University of Washington, Seattle, Washington, USA ${ }^{175}$ Department of Psychiatry, Washington University in St. Louis, School of Medicine, Saint Louis, Missouri, USA ${ }^{176}$ Department of Mathematics, Washington 
University in St. Louis, School of Medicine, Saint Louis, Missouri, USA ${ }^{177}$ MRC-HPA Centre for Environment and Health, Imperial College London, London, UK ${ }^{178}$ Centre of Medical Systems Biology, Erasmus University Medical Center, Rotterdam, The Netherlands

\section{Abstract}

Numerous genetic loci influence systolic blood pressure (SBP) and diastolic blood pressure (DBP) in Europeans ${ }^{1-3}$. We now report genome-wide association studies of pulse pressure (PP) and mean arterial pressure (MAP). In discovery $(\mathrm{N}=74,064)$ and follow-up studies $(\mathrm{N}=48,607)$, we identified at genome-wide significance $\left(P=2.7 \times 10^{-8}\right.$ to $\left.P=2.3 \times 10^{-13}\right)$ four novel PP loci (at $4 \mathrm{q} 12$ near CHIC2/PDGFRAI, 7q22.3 near PIK3CG, 8q24.12 in NOV, 11q24.3 near ADAMTS-8), two novel MAP loci (3p21.31 in MAP4, 10q25.3 near $A D R B 1$ ) and one locus associated with both traits (2q24.3 near $F I G N$ ) which has recently been associated with SBP in east Asians. For three of the novel PP signals, the estimated effect for SBP was opposite to that for DBP, in contrast to the majority of common SBP- and DBP-associated variants which show concordant effects on both traits. These findings indicate novel genetic mechanisms underlying blood pressure variation, including pathways that may differentially influence SBP and DBP.

High blood pressure is a major risk factor for coronary heart disease and stroke ${ }^{4}$. Large genome-wide association studies in Europeans have reported 29 novel loci for systolic and diastolic blood pressure (SBP and DBP) where alleles have effect sizes of up to $0.5-1 \mathrm{~mm}$ $\mathrm{Hg}^{1-3}$. Even small increments in blood pressure levels have important effects on cardiovascular morbidity and mortality at the population level ${ }^{5}$. We undertook a genomewide association study of two further blood pressure phenotypes, pulse pressure (PP, the difference between SBP and DBP), a measure of stiffness of the main arteries, and mean arterial pressure (MAP), a weighted average of SBP and DBP. Both PP and MAP are predictive of hypertension ${ }^{6}$ and cardiovascular disease ${ }^{7-9}$.

This study was undertaken by the International Consortium of Blood Pressure GenomeWide Association Studies (ICBP-GWAS) which aims to further the understanding of the genetic architecture underlying blood pressure. The initial publication by this consortium ${ }^{1}$ studied SBP and DBP with discovery GWAS among 69,395 people and a combined sample of $\sim 200,000$ Europeans. The two blood pressure phenotypes reported here, namely PP and MAP, were not previously analysed. All but one study that was included in the discovery GWAS of the study of SBP and DBP were included in the discovery GWAS stage of this study. In addition, a further 6 studies not included in the previous study ${ }^{1}$ were included here bringing our discovery GWAS sample size to 74,064 .

We first conducted a genome-wide association meta-analysis of PP and MAP in 74,064 individuals of European ancestry from 35 studies (Supplementary Table 1A). Genotypes were imputed using HapMap. To account for effects of anti-hypertensive treatments, we imputed underlying SBP and DBP by adding a constant to ach $^{2,3}$. Associations were adjusted for age, age ${ }^{2}$, sex and body mass index. We combined results across studies using an inverse variance weighted meta-analysis and, to correct for residual test statistic inflation, applied genomic control (GC) both to study-level association statistics and to the metaanalysis $\left(\lambda_{\mathrm{GC}}=1.08 \text { for PP, } \lambda_{\mathrm{GC}}=1.12 \text { for MAP }\right)^{10}$. The QQ plots show an excess of extreme values largely accounted for by a modest number of genomic regions (Supplementary Figures 1 (a) - (b)). Independent follow-up analyses were performed in 48,607 individuals of European ancestry (Online Methods and Supplementary Note).

SNPs in 12 regions showed genome-wide significant association $\left(P<5 \times 10^{-8}\right)$ with either PP or MAP in our discovery data (Stage 1) (Supplementary Figures 1 (c) $-($ d)), including two 
novel regions for PP (7q22.3 near PIK3CG, $P=1.2 \times 10^{-10}$ and 11q24.3 near ADAMTS8, $P=8.5 \times 10^{-11}$; Table 1$)$ and 10 regions previously associated with SBP and DBP (Supplementary Table 2A for PP, Supplementary Table 2B for MAP) ${ }^{1-3}$. For follow-up in a series of independent cohorts we selected 99 SNPs comprising those with $P<1 \times 10^{-5}$ for either PP or MAP and SNPs reported in recent large genome-wide association studies of SBP and $\mathrm{DBP}^{1-3}$ to evaluate their effects on PP and MAP (Stage 2: Online Methods, Supplementary Note).

After meta-analysis of the Stage 1 and Stage 2 data (Supplementary Table 2C), the two novel regions showing genome-wide association with PP after Stage 1 (near PIK3CG and near ADAMTS8) remained genome-wide significant. In addition, we found genome-wide significant associations for SNPs at two further novel loci for PP (at 4q12 near CHIC2/ PDGFRA and 8q24.12 in NOV), two novel loci for MAP (3p21.31 in MAP4, 10q25.3 near $A D R B 1$ ), and one locus for both traits (2q24.3 near FIGN) (Table 1 and Figure 1) which has not previously shown an association with SBP or DBP in Europeans but which has recently been associated with SBP in east Asians (see Supplementary Note) ${ }^{11}$. Forest plots of the Stage 1 effect sizes and standard errors are shown in Supplementary Figure 2. The novel signals for MAP were strongly associated with both SBP and DBP $\left(P=7.7 \times 10^{-7}\right.$ to $P=1.8 \times 10^{-12}$ ), reflecting the high inter-correlations among these three blood pressure traits $^{12,13}$. For the sentinel SNPs in three of the novel PP loci, the estimated effects on SBP were in the opposite direction to the effects on DBP (Table 1, Figure 2, Supplementary Tables 2D and 2E). Our findings show that analyses of PP and MAP reveal loci influencing blood pressure phenotypes which may not be detectable by studying SBP and DBP separately. Identification of novel genetic associations could help inform understanding about possible distinct mechanisms underlying relationships of PP with vascular risk ${ }^{14,15}$.

Five additional loci for PP and 19 loci for MAP reaching genome-wide significance $\left(P<5 \times 10^{-8}\right.$, Stage 1 and Stage 2 combined) were recently shown to be associated with SBP/ $\mathrm{DBP}^{1-3}$ (Supplementary Tables $2 \mathrm{~A}$ and $2 \mathrm{~B}$ ). We used sentinel SNPs from both the novel and known regions showing genome-wide significant associations with PP or MAP in the combined Stage 1 and 2 data to create weighted risk scores for: i) PP (10 independent SNPs) and; ii) MAP (22 SNPs) (Supplementary Table 2F). We studied the associations of both risk scores with hypertension and blood pressure related outcomes including coronary heart disease, heart failure, stroke, echocardiographic measures of left ventricular structure, pulse wave velocity, renal function and renal failure. Adjusting for multiple testing for the 12 traits evaluated $\left(P=0.05 / 12=4.1 \times 10^{-3}\right)$, the PP SNP risk score was associated with prevalent hypertension $\left(P=7.9 \times 10^{-6}\right)$, incident stroke $\left(P=4.9 \times 10^{-4}\right)$ and coronary heart disease $\left(P=4.3 \times 10^{-4}\right)$, and the MAP SNP risk score was associated with hypertension $\left(P=5.1 \times 10^{-16}\right)$, coronary heart disease $\left(P=4.0 \times 10^{-20}\right)$, stroke $(P=0.0019)$ and left ventricular wall thickness $\left(P=2.1 \times 10^{-4}\right)$ (Supplementary Table 3A), confirming the clinical relevance of these measures of blood pressure phenotype ${ }^{8,9}$. For a range of blood pressure related outcomes (see Supplementary Note), we compared $P$ values for the PP risk score and a series of 1000 permutations of SBP risk scores, each based on 10 of the 26 blood pressure SNPs associated with SBP but not PP, constraining the selection of SNPs to have similar sized effects for SBP as those of the 10 PP SNPs. The PP risk score had a significantly $(P<0.05)$ greater association with risk of ischemic stroke than the SBP risk score (Supplementary Note and Supplementary Table 3B).

None of the genes in the identified novel regions is a strong candidate for blood pressure regulation, although several are implicated in mechanisms that may influence blood pressure. The most significant association with $\mathrm{PP}$ is within a putative mRNA clone (AF086203) spanning 13.7kb at 7q22.3, 94kb upstream of PIK3CG (rs17477177, $P=2.3 \times 10^{-13}$, Table 1 and Figure 1a). PIK3CG encodes the phosphoinositide-3-kinase, 
catalytic, gamma polypeptide protein which phosphorylates phosphoinositides and modulates extracellular signals. This region was earlier associated with mean platelet volume, platelet count, and platelet aggregation ${ }^{16-18}$, but the sentinel SNPs reported in those studies are independent of SNP rs17477177 reported here $\left(\mathrm{r}^{2}<0.01\right)$. Mice lacking the catalytic subunit of PI3K $\gamma$ have shown resistance to SBP-lowering effects of betaadrenergic receptor agonists ${ }^{19}$; $\mathrm{PI} 3 \mathrm{~K} \gamma$ activity is increased in the failing human heart and associated with down-regulation of beta-adrenergic receptors in the plasma membrane ${ }^{20}$. The second locus for PP located at $11 \mathrm{q} 24.3$ spans $35.5 \mathrm{~kb}$ with the top-ranking SNP (rs11222084, $P=1.9 \times 10^{-11}$, Figure 1b) lying 1.6kb downstream of ADAMTS-8. This gene is highly expressed in macrophage-rich areas of human atherosclerotic plaques and may affect extracellular matrix remodeling ${ }^{21}$. The third locus for PP spans $28.5 \mathrm{~kb}$ at $8 \mathrm{q} 24.12$ with the sentinel SNP (rs2071518, $P=3.7 \times 10^{-9}$, Figure 1c) located in the 3'UTR of $N O V$ which encodes the nephroblastoma overexpressed (CCN3) protein, associated with angiogenesis, proliferation, and inhibition of vascular smooth muscle cell growth and migration ${ }^{22}$, and with reduced neointimal thickening in mice null for $\mathrm{CCN}^{23}$. Mice with mutations in $\mathrm{NOV}$ that truncate the NOV protein exhibit abnormal cardiac development ${ }^{24}$. Of the genes evaluated for expression in human aortic samples at the novel PP loci, NOV showed by far the highest expression levels (Supplementary Note and Supplementary Figure 3). The fourth locus for PP is $4 \mathrm{q} 12$ with the top-ranking SNP (rs871606, $P=1.3 \times 10^{-8}$, Figure 1d) located $76.7 \mathrm{~kb}$ downstream of $C H I C 2$ which encodes a cysteine-rich hydrophobic domain containing protein associated with acute myeloid leukaemia ${ }^{25}$. This SNP is located $296 \mathrm{~kb}$ upstream of PDGFRA which encodes platelet-derived growth factor receptor alpha, a cell surface receptor for members of the platelet-derived growth factor family involved in kidney development. Variants in PDGFRA have been associated with red blood cell count and other haematological indices ${ }^{26}$ but are independent $\left(\mathrm{r}^{2}<0.3\right)$ of rs871606.

For MAP we identified two novel loci. The first locus for MAP is at $10 \mathrm{q} 25.3,22.3 \mathrm{~kb}$ upstream of $A D R B 1$ (rs2782980, $P=2.5 \times 10^{-9}$, Figure 1e). $A D R B 1$ encodes the beta-1adrenergic receptor, which mediates the effects of the stimulatory G protein and cAMP/ protein kinase A pathway to increase heart rate and myocardial contraction. Polymorphisms in this gene have been associated with resting heart rate, response to beta-blockers ${ }^{27}$, and hypertension ${ }^{28}$. $A D R B 1$ knockout mice have no difference in heart rate or blood pressure compared with the wild type but do exhibit a significant reduction in the response of both phenotypes to catecholamines ${ }^{29}$. SNP rs2782980 is associated with expression of an $A D R B 1$ transcript in brain tissue (Supplementary Note and Supplementary Figure 4A). The second locus for MAP spans over 300kb at 3p21.31 with the top-ranking SNP (rs319690, $P=2.7 \times 10^{-8}$, Figure 1f) lying within an intron of the microtubule associated protein 4 gene, MAP4. Coating of microtubules by MAP4 may inhibit beta adrenergic receptor recycling and number, as seen in cardiac hypertrophy and failure ${ }^{30}$. MAP4 was detectably expressed in human aortic samples (Supplementary Note and Supplementary Figure 3).

The locus associated both with PP (SNP rs13002573, $P=1.8 \times 10^{-8}$, Figure $1 \mathrm{~g}$ ) and MAP (rs1446468, $P=6.5 \times 10^{-12}$, Figure $1 \mathrm{~h}$ ) is in an intergenic region spanning $\sim 280 \mathrm{~kb}$ at $2 \mathrm{q} 24.3$. Although the two signals are $\sim 50 \mathrm{~kb}$ apart and statistically independent $\left(\mathrm{r}^{2}=0.075\right)$, rs 13002573 is highly correlated $\left(r^{2}=1\right.$ in HapMap CEU population, $\mathrm{r}^{2}=0.87$ in HapMap JPT $+\mathrm{CHB}$ ) with rs 16849225 which has recently been reported as showing association with SBP in a GWAS of 19,608 subjects of east Asian origin with follow-up in a further 30,765 individuals (combined result: $P=3.5 \times 10^{-11}$ ) ${ }^{11}$ (see Supplementary Note). In our combined dataset in 116,998 Europeans, the association $P$ value for rs 13002573 with SBP was $P=3.25 \times 10^{-7}$. The top PP SNP lies $\sim 320 \mathrm{~kb}$ upstream of $F I G N$ and $\sim 430 \mathrm{~kb}$ downstream of GRB14 (growth factor receptor-bound protein 14). Relatively little is known regarding $F I G N$ (fidgetin). 
We report six novel loci associated with PP and MAP based on genome-wide discovery and follow-up in over $~ 120,000$ individuals, and a further locus (near FIGN) not previously reported in Europeans. Our results expand knowledge of the genetic architecture of blood pressure and PP regulation and may give clues as to possible novel targets for blood pressure therapies.

\section{Online Methods}

Pulse pressure was defined as systolic minus diastolic pressure and MAP as $2 / 3$ diastolic plus $1 / 3$ systolic pressure. A two-staged analysis was used to discover genes associated with $\mathrm{PP}$ and MAP.

\section{Stage 1 samples and analyses}

Stage 1 was a meta-analysis of directly genotyped and imputed SNPs from population-based or control samples from case-control studies, in the International Consortium of Blood Pressure Genome-wide Association Studies (ICBP-GWAS). The characteristics of the 35 studies, including demographics, genotyping arrays, quality control filters and statistical analysis methods used are listed in Supplementary Tables 1A and 1B. Imputation of allele dosage of ungenotyped SNPs in HapMap CEU v21a or v22 was carried out by each of the studies using $\mathrm{MACH}^{31}$, IMPUTE ${ }^{32}$ or BIMBAM ${ }^{33}$ with parameters and pre-imputation filters as specified in Supplementary Table 1B. SNPs were excluded from analysis if the study-specific imputation quality (r2.hat in MACH or .info in IMPUTE) was <0.3. In total, up to 2652054 genotyped or imputed autosomal SNPs were analyzed. Full details of the models, methods, and corrections for antihypertensive treatment are provided in the Supplementary methods. All analyses assumed an additive genetic model and were adjusted for sex, age, age $^{2}$, body mass index and ancestry principal components. In related individuals, regression methods that account for relatedness were applied. All study-specific effect estimates and coded alleles were oriented to the forward strand of the HapMap release 22 with the alphabetically higher allele as the coded allele. To capture loss of power due to imperfect imputation, we estimated "N effective" as the sum of the study-specific products of the imputation quality metric and the sample size. No filtering on minor allele frequency was done. Genomic control was carried out on study-level data and inverse variance weighting was used for meta-analysis of Stage 1 . The meta-analysis results were subject to genomic control. Lambda estimates are given in Supplementary Table 1A.

\section{Selection of SNPs for Stage 2}

We aimed in Stage 2 to follow up SNPs which had evidence of association with PP or MAP and, for completeness, to evaluate the effects on PP and MAP of SNPs reported in recent large genome-wide association studies of SBP and DBP ${ }^{1-3}$. All SNPs with $P<1 \times 10^{-5}$ for association with either PP or MAP (or both) were divided into independent regions based on LD and the most significant SNP was selected from each region. Within the FIGN region, different SNPs were associated with PP and with MAP and both SNPs were followed up in Stage 2. For SNPs with an N effective $<75 \%$ of total N, a proxy was also included if it had $P$ $<1 \times 10^{-5}$ and an $\mathrm{r}^{2}>0.6$ with the top SNP (this occurred for one SNP). For all regions that had previously shown association with SBP or $\mathrm{DBP}^{1-3}$, the sentinel SNP for PP and MAP and the previously reported SNP for SBP and DBP were followed up. In all, 99 SNPs were followed up in Stage 2 (Supplementary Note), comprising: 44 SNPs from 22 loci with PP or MAP associations $\left(P<1 \times 10^{-5}\right)$ in Stage 1 data and with previously reported SBP or DBP associations; 47 SNPs from 45 loci with PP or MAP associations $\left(P<1 \times 10^{-5}\right)$ in Stage 1 data only and; 8 SNPs from 7 loci with previously reported SBP or DBP associations and no association $\left(P<1 \times 10^{-5}\right)$ with PP or MAP in the Stage 1 data. 
Stage 2

The characteristics of the Stage 2 studies, including the genotyping and imputation approaches, are described in Supplementary Tables 1A and 1B and the details of corrections for treatment described in the Supplementary Note. For the 99 SNPs selected for follow-up, the Stage 2 studies followed the analysis approach adopted in the Stage 1 analyses. Metaanalysis was done using the inverse variance weights method.

\section{Pooled analysis of first and second stage samples}

Meta-analysis from stages 1 and 2 was conducted using inverse variance weighting and genomic control applied. A threshold of $5 \times 10^{-8}$ was taken for genome-wide significance.

\section{Calculation of risk scores}

We calculated risk scores based on the most significantly associated SNP from all regions which were genome-wide significant after meta-analysis of Stages 1 and 2 for i) PP (10 SNPs) and ii) MAP (22 SNPs) (Supplementary Table 2F). Each risk score was constructed using an approach described in the Supplementary Note and was tested for association with hypertension, coronary artery disease, stroke, hypertension, chronic kidney disease, heart failure, microalbuminuria, and with continuous traits left ventricular mass, left ventricular wall thickness, pulse wave velocity, serum creatinine, eGFR and urinary albumin:creatinine ratio (Supplementary Table 3).

\section{Additional analyses}

Identification of potentially functional SNPs in LD with the reported sentinel SNPs, eQTL analyses and expression analyses in human aortic samples were also carried out as discussed in the Supplementary Note and Supplementary Figures 3 and 4.

\section{Supplementary Material}

Refer to Web version on PubMed Central for supplementary material.

\section{Acknowledgments}

A number of the participating studies and authors are members of the CHARGE and Global BPgen consortia. Many funding mechanisms by NIH/NHLBI, European, and private funding agencies contributed to this work and a full list is provided in the Supplementary Note.

\section{Contributions}

ICBP-GWAS PP/MAP Working and Writing Sub-Group (alphabetical order) M.J.C., P.E. (co-chair), T.J., P.B.M., P.F.O'R., M.D.T. (co-chair), C.M.V. (co-chair), G.C.V., L.V.W. ICBP-GWAS Steering Committee (alphabetical order) G.R.A., M.Bochud, M.Boehnke, MJ.C. (co-chair), A.C., G.B.E., P.E., T.B.H., M-R.J, A.D.J., T.J., M.G.L., L.L., D.L. (co-chair), P.B.M.(co-chair), C.N-C. (co-chair), B.M.P., K.M.R., A.V.S., M.D.T., C.M.V, G.C. V. Analysis L.V.W., G.C.V., P.F.O'R., T.J. Expression analyses V.E., P.H., A.D.J., D.L., J.H.L., C.P.N, A.Plump, P.A.C't H., K.W.V. Cohort contributions (alphabetical order): Study concept/design: AGES: T.A., V.G., T.B.H., L.L., A.V.S., AortaGen Consortium: G.F.M., ARIC: E.B., A.C., S.K.G., ASPS: H.Schmidt, R.S., BLSA: L.F., B58C-T1DGC: D.P.S., B58C-WTCCC: D.P.S., BHS: L.J.P., CardioGram Consortium: N.J.S., C4D Consortium: R.Clarke, R.Collins, CHS: J.C.B., N.L.G., B.M.P., K.M.R., K.D.T., CHARGE Consortium Heart Failure Working Group: N.L.S., CoLaus: V.M., P.Vollenweider, G.Waeber, CROATIA-Korcula: C.H., CROATIA-Split: M.Boban, I.R., CROATIA-Vis: A.F.W., DeCode Genetics: H.H., K.S., G.T., U.T., DGI controls: D.A., 
L.G., C.N-C., ENGAGE: J.E., I.R.K., EGCUT: H.A., A.M., EPIC: K-T.K., ERF: B.A.O., Fenland: N.J.W., FUSION: M.Boehnke, F.S.C., R.N.B., J.T., INGI CARL: A.P.d'A., P.Gasparini, INGI-FVG: A.P.d'A., P.Gasparini, INCHIANTI: S.Bandinelli., Y.M., KORA S3: C.G., M.Laan, E.O., KORA F4: T.M, H-E.W., LifeLines: R.P.S., M.M.V., LOLIPOP: J.C.C., P.E., J.S.K., LBC1921/LBC1936: I.J.D., J.M.S., MICROS: A.Pfeufer, MESA: X.G., W.P., MIGen controls: O.M., C.J.O., V.S., D.Siscovick, NESDA: B.W.P., H.Snieder, NEURO-CHARGE Consortium: M.Breteler M.Fornage, NFBC1966: M-R.J, P.Z, NSPHS: U.B.G., S.E.H., NTR: D.I.B., E. J.C. deG., ORCADES: H.C., J.F.W., PROCARDIS controls: M.Farrall, A.Hamsten, J.F.P., H.W., PROSPER/PHASE: B.B., J.W.J., D.Stott, RSI/RSII/RSIII: A.Hofman, C. M.V., J.C.M.W., SardiNIA: G.A., M.U., SHIP: M.D., H.K.K., R.R., U.V., H.V., SUVIMAX: P.Gilan, S.Hercberg, P.M., TwinsUK: T.D.S., WGHS: P.M.R., YFS: M.K., T.L., O.T.R., J.V. Phenotype data acquisition/QC: AGES: T.A., V.G., T.B.H., L.L., ARIC: A.C., S.K.G., A.C.M, D.C.R., ASPS: M.Loitfelder, R.S., BLSA: S.N., B58C-T1DGC: D.P.S., B58C-WTCCC: D.P.S., BHS: J.P.B., J.H., C4D Consortium: R.Clarke, R.Collins, J.C.H., CHS: B.M.P., CoLaus: M.Bochud, V.M., P.Vollenweider, CROATIA-Korcula: C.H., O.P., CROATIA-Split: M.Boban, I.R., DGI controls: L.G., C.N-C., EGCUT: H.A., A.K., A.M., M-L.T., EPIC: N.J.W., Fenland: N.J.W., FHS: S.-J.H., M.G.L., D.L., R.S.V., T.J.W., FUSION: J.T., INGI CARL: A.F., F.F., P.Gasparini, S.U., INGI FVG: A.F., F.F., P.Gasparini, S.U., INGI-Val Borbera: C.Masciullo, C.S., D.T., INCHIANTI: A.M.C., KORA S3: C.G., KORA F4: A.D., LifeLines: M.M.V., LOLIPOP: J.C.C., J.S.K., J.S., LBC1921/LBC1936: I.J.D., L.M.L., J.M.S., MICROS: M.Facheris, A.Pfeufer, MESA: X.G., W.P., MIGen controls: G.L., O.M., C.J.O., V.S., D.Siscovick, NESDA: : X.Lu, I.M.N., B.W.P., H.Snieder, NEURO-CHARGE Consortium: M.Breteler, S.D., A.L.D., M.Fornage, NFBC1966: P.E., M-R.J., J.Laitinen, A.Pouta, P.Z., NSPHS: J.A.C., U.B.G., S.E.H., P.J.T., NTR: D.I.B., E.J.C.deG., G.Willemsen, ORCADES: S.H.W., J.F.W., PROCARDIS controls: J.F.P., PROSPER/ PHASE: D.Stott, S.T., RSI/RSII/RSIII: F.U.S.M.R., E.J.G.S., C.M.V., G.C.V., J.C.M.W., SardiNIA: M.O., M.U., SHIP: M.D., R.R., H.V., SUVIMAX: P.Gilan, M.Lathrop, TwinsUK: T.D.S., WGHS: D.I.C., A.N.P., YFS: M.K., T.L., O.T.R., J.V. Genotype data acquisition/QC: AGES: A.V.S., ARIC: A.C., G.B.E., S.K.G., A.C.M., D.C.R, G.S., ASPS: P.Gider, H. Schmidt, M.Z., BLSA: D.Hernandez, B58C-T1DGC: S.Heath, W.L.McA., B58C-WTCCC: W.L.McA., BHS: J.P.B., R.J.W., C4D Consortium: J.C.H., H.O., CHS: J.C.B., N.L.G., K.D.T., CoLaus: V.M., P.Vollenweider, CROATIA-Korcula: C.H., O.P., CROATIA-Split: I.R., CROATIA-Vis: V.V., DGI controls: D.A., B.F.V., EGCUT: T.E., T.H., EPIC: N.J.W., Fenland: R.J.F.L., J.Luan, N.J.W., FHS: S.-J.H., M.G.L., FUSION: F.S.C., INGI CARL: A.P.d'A., INGI FVG: A.P.d'A., INGI Val Borbera: C.Masciullo, C.S., D.T., INCHIANTI: A.S., KORA S3: C.G., M.Laan, E.O., KORA F4: T.M, H-E.W., LifeLines: B.Z.A., LOLIPOP: J.C.C., J.S.K., J.S., W.Z., LBC1921/LBC1936: G.D., I.J.D., MICROS: I.P., MESA: X.G., MIGen controls: G.L., O.M., C.J.O., V.S., D.S., NESDA: J.F., X.Lu, I.M.N., B.W.P., H.Snieder, NFBC1966: P.E., M-R.J., J.Laitinen, P.Z., NSPHS: J.P., P.J.T., NTR: D.I.B., E.J.C.deG., J-J.H., G.Willemsen, ORCADES: H.C., J.F.W., PROCARDIS controls: A.G., J.F.P., PROSPER/PHASE: S.T., RSI/RSII/RSIII: F.R., A.G.U., SardiNIA: G.A., SHIP: H.K.K., U.V., H.V., SUVIMAX: S.Heath, M.Lathrop, TwinsUK: M.M., S-Y.S, N.S., F.Z., WGHS: P.M.R., YFS: T.L., O.T.R. Data analysis: AGES: T.A., A.V.S, ARIC: A.C., G.B.E., A.C.M., V.P., D.C.R, G.S., ASPS: P.Gider, H. Schmidt, M.Z., BLSA: T.T. B58C-T1DGC: D.P.S., B58C-WTCCC: D.Hadley, D.P.S., BHS: W.A.M., L.J.P., R.J.W., C4D Consortium: J.C.H., H.O., CHS: J.C.B., N.L.G., K.M.R., CoLaus: S.Bergmann, M.Bochud, T.J., CROATIA-Korcula: C.H., O.P., CROATIA-Split: C.H., CROATIA-Vis: V.V., DGI controls: P.A., C.N-C., B.F.V., EchoGen Consortium: J.F.F., EGCUT: T.E., T.H., ENGAGE: M.P., EPIC: I.B., R.J.F.L., N.J.W., J.H.Z., ERF: A.C.J.W.J., Y.A., Fenland: R.J.F.L, J.Luan., FHS: S.-J.H., M.G.L., FUSION: A.U.J., INGI CARL: N.P., INGI FVG: N.P., INGI Val Borbera: T.C., G.P., C.S., D.T., 
KORA S3: S.E., S.S., KORA F4: B.K., LifeLines: B.Z.A., LOLIPOP: J.C.C., J.S.K., X.Li, J.S., W.Z., LBC1921/LBC1936: L.M.L., MICROS: F.D-G.M., MESA: X.G., W.P., MIGen controls: G.L., NESDA: J.F., X.Lu., NEURO-CHARGE Consortium: S.D., A.L.D, M.Fornage, NFBC1966: P.F.O’R., NSPHS: J.A.C., W.I., NTR: J-J.H., ORCADES: P.N., S.H.W., J.F.W., PROCARDIS controls: M.Farrall, A.G., J.F.P., PROSPER/PHASE: J.W.J., S.T., RSI/RSII/RSIII: N.A., S.K., C.M.V., G.C.V., SardiNIA: J.L.B-G., SHIP: U.V., SUVIMAX: T.J., P.M., TwinsUK: N.S., F.Z., WGHS: D.I.C., L.M.R., YFS: T.L., O.T.R.

\section{References}

1. Ehret G, et al. Genetic Variants in Novel Pathways Influence Blood Pressure and Cardiovascular Disease Risk. Nature. 2011

2. Levy D, et al. Genome-wide association study of blood pressure and hypertension. Nat Genet. 2009; 41:677-87. [PubMed: 19430479]

3. Newton-Cheh C, et al. Genome-wide association study identifies eight loci associated with blood pressure. Nat Genet. 2009; 41:666-76. [PubMed: 19430483]

4. Lawes CM, et al. Blood pressure and the global burden of disease 2000. Part II: estimates of attributable burden. J Hypertens. 2006; 24:423-30. [PubMed: 16467640]

5. Rose, G. Strategies of prevention: the individual and the population. In: Marmot, M.; E, P., editors. Coronary heart disease epidemiology: From aetiology to Public health. Oxford University Press; Oxford: 2005. p. 631-41.

6. Domanski MJ, et al. Independent prognostic information provided by sphygmomanometrically determined pulse pressure and mean arterial pressure in patients with left ventricular dysfunction. $\mathrm{J}$ Am Coll Cardiol. 1999; 33:951-8. [PubMed: 10091821]

7. Domanski M, et al. Pulse pressure and cardiovascular disease-related mortality: follow-up study of the Multiple Risk Factor Intervention Trial (MRFIT). Jama. 2002; 287:2677-83. [PubMed: 12020303]

8. Franklin SS, et al. Single versus combined blood pressure components and risk for cardiovascular disease: the Framingham Heart Study. Circulation. 2009; 119:243-50. [PubMed: 19118251]

9. Lewington S, Clarke R, Qizilbash N, Peto R, Collins R. Age-specific relevance of usual blood pressure to vascular mortality: a meta-analysis of individual data for one million adults in 61 prospective studies. Lancet. 2002; 360:1903-13. [PubMed: 12493255]

10. Devlin B, Roeder K. Genomic control for association studies. Biometrics. 1999; 55:997-1004. [PubMed: 11315092]

11. Kato N, et al. Meta-analysis of genome-wide association studies identifies common variants associated with blood pressure variation in east Asians. Nat Genet. May 15.2011 published online.

12. Sesso HD, et al. Systolic and diastolic blood pressure, pulse pressure, and mean arterial pressure as predictors of cardiovascular disease risk in Men. Hypertension. 2000; 36:801-7. [PubMed: 11082146]

13. Darne B, Girerd X, Safar M, Cambien F, Guize L. Pulsatile versus steady component of blood pressure: a cross-sectional analysis and a prospective analysis on cardiovascular mortality. Hypertension. 1989; 13:392-400. [PubMed: 2522417]

14. Blacher J, Safar ME. Large-artery stiffness, hypertension and cardiovascular risk in older patients. Nat Clin Pract Cardiovasc Med. 2005; 2:450-5. [PubMed: 16265585]

15. Dart AM, Kingwell BA. Pulse pressure--a review of mechanisms and clinical relevance. J Am Coll Cardiol. 2001; 37:975-84. [PubMed: 11263624]

16. Johnson AD, et al. Genome-wide meta-analyses identifies seven loci associated with platelet aggregation in response to agonists. Nat Genet. 2010; 42:608-13. [PubMed: 20526338]

17. Soranzo N, et al. A novel variant on chromosome 7q22.3 associated with mean platelet volume, counts, and function. Blood. 2009; 113:3831-7. [PubMed: 19221038]

18. Soranzo N, et al. A genome-wide meta-analysis identifies 22 loci associated with eight hematological parameters in the HaemGen consortium. Nat Genet. 2009; 41:1182-90. [PubMed: 19820697] 
19. Oudit GY, et al. Phosphoinositide 3-kinase gamma-deficient mice are protected from isoproterenol-induced heart failure. Circulation. 2003; 108:2147-52. [PubMed: 12963636]

20. Perrino C, et al. Dynamic regulation of phosphoinositide 3-kinase-gamma activity and betaadrenergic receptor trafficking in end-stage human heart failure. Circulation. 2007; 116:2571-9. [PubMed: 17998459]

21. Wagsater D, et al. ADAMTS-4 and -8 are inflammatory regulated enzymes expressed in macrophage-rich areas of human atherosclerotic plaques. Atherosclerosis. 2008; 196:514-22. [PubMed: 17606262]

22. Ellis PD, Chen Q, Barker PJ, Metcalfe JC, Kemp PR. Nov gene encodes adhesion factor for vascular smooth muscle cells and is dynamically regulated in response to vascular injury. Arterioscler Thromb Vasc Biol. 2000; 20:1912-9. [PubMed: 10938011]

23. Shimoyama T, et al. CCN3 inhibits neointimal hyperplasia through modulation of smooth muscle cell growth and migration. Arterioscler Thromb Vasc Biol. 2010; 30:675-82. [PubMed: 20139355]

24. Heath E, et al. Abnormal skeletal and cardiac development, cardiomyopathy, muscle atrophy and cataracts in mice with a targeted disruption of the Nov (Ccn3) gene. BMC Dev Biol. 2008; 8:18. [PubMed: 18289368]

25. Cools J, et al. Fusion of a novel gene, BTL, to ETV6 in acute myeloid leukemias with a t $(4 ; 12)$ (q11-q12;p13). Blood. 1999; 94:1820-4. [PubMed: 10477709]

26. Kamatani Y, et al. Genome-wide association study of hematological and biochemical traits in a Japanese population. Nat Genet. 2010; 42:210-5. [PubMed: 20139978]

27. Dorn GW 2nd. Adrenergic signaling polymorphisms and their impact on cardiovascular disease. Physiol Rev. 2010; 90:1013-62. [PubMed: 20664078]

28. Kitsios GD, Zintzaras E. Synopsis and data synthesis of genetic association studies in hypertension for the adrenergic receptor family genes: the CUMAGAS-HYPERT database. Am J Hypertens. 2010; 23:305-13. [PubMed: 20044737]

29. Rohrer DK, Chruscinski A, Schauble EH, Bernstein D, Kobilka BK. Cardiovascular and metabolic alterations in mice lacking both beta1- and beta2-adrenergic receptors. J Biol Chem. 1999; 274:16701-8. [PubMed: 10358009]

30. Cheng G, Qiao F, Gallien TN, Kuppuswamy D, Cooper Gt. Inhibition of beta-adrenergic receptor trafficking in adult cardiocytes by MAP4 decoration of microtubules. Am J Physiol Heart Circ Physiol. 2005; 288:H1193-202. [PubMed: 15528234]

31. Li Y, Abecasis GR. Mach 1.0: Rapid haplotype reconstruction and missing genotype inference. Am J Hum Genet S. 2006; 79:2290.

32. Marchini J, Howie B, Myers S, McVean G, Donnelly P. A new multipoint method for genomewide association studies by imputation of genotypes. Nat Genet. 2007; 39:906-13. [PubMed: 17572673]

33. Servin B, Stephens M. Imputation-based analysis of association studies: candidate regions and quantitative traits. PLoS Genet. 2007; 3:e114. [PubMed: 17676998] 


$$
\text { PP rs17477177 }
$$

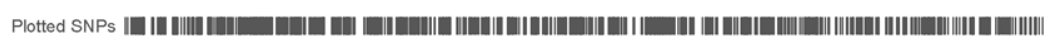

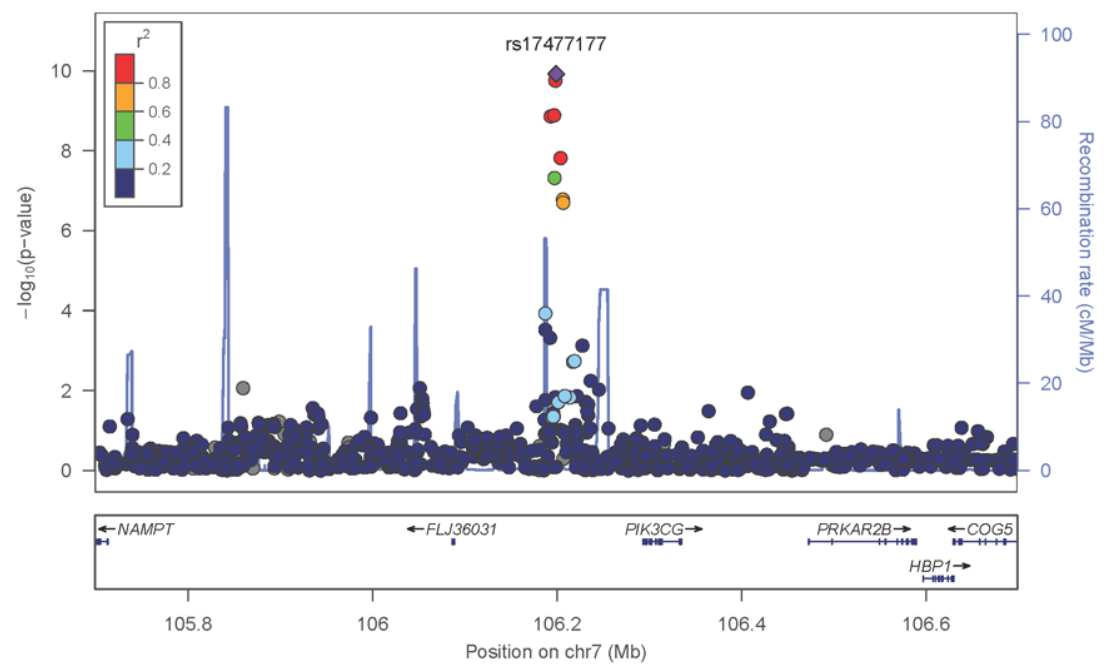

PP rs11222084

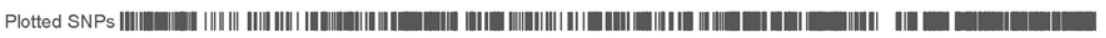

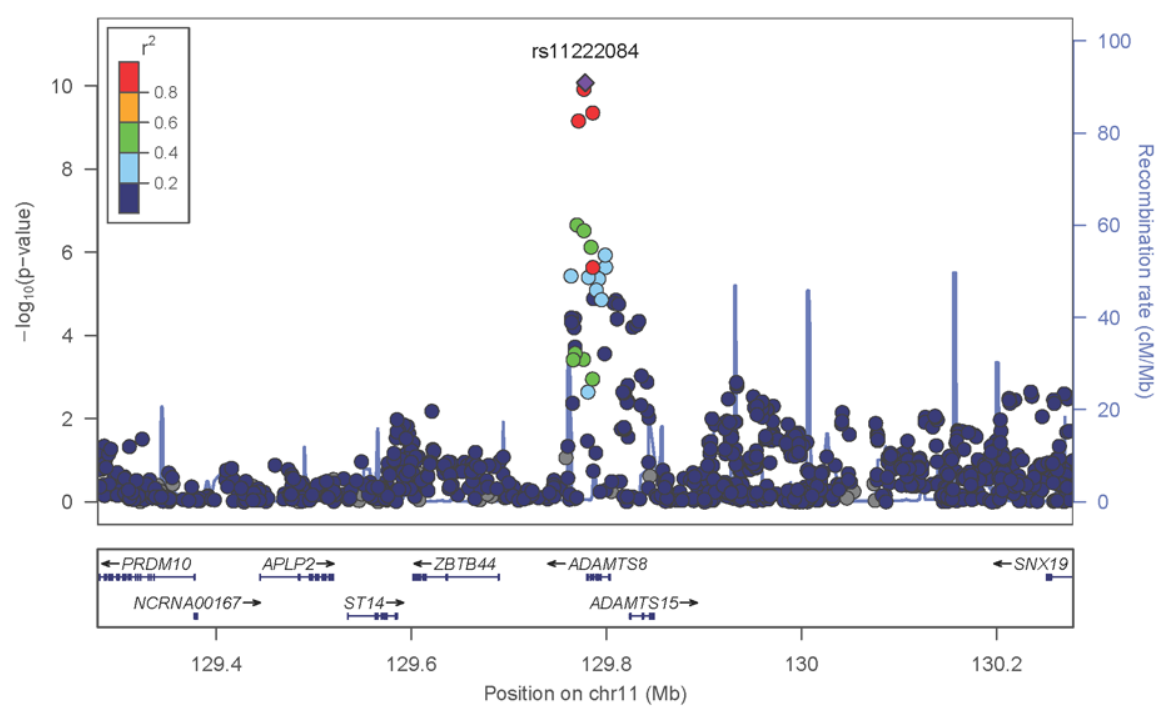


PP rs2071518
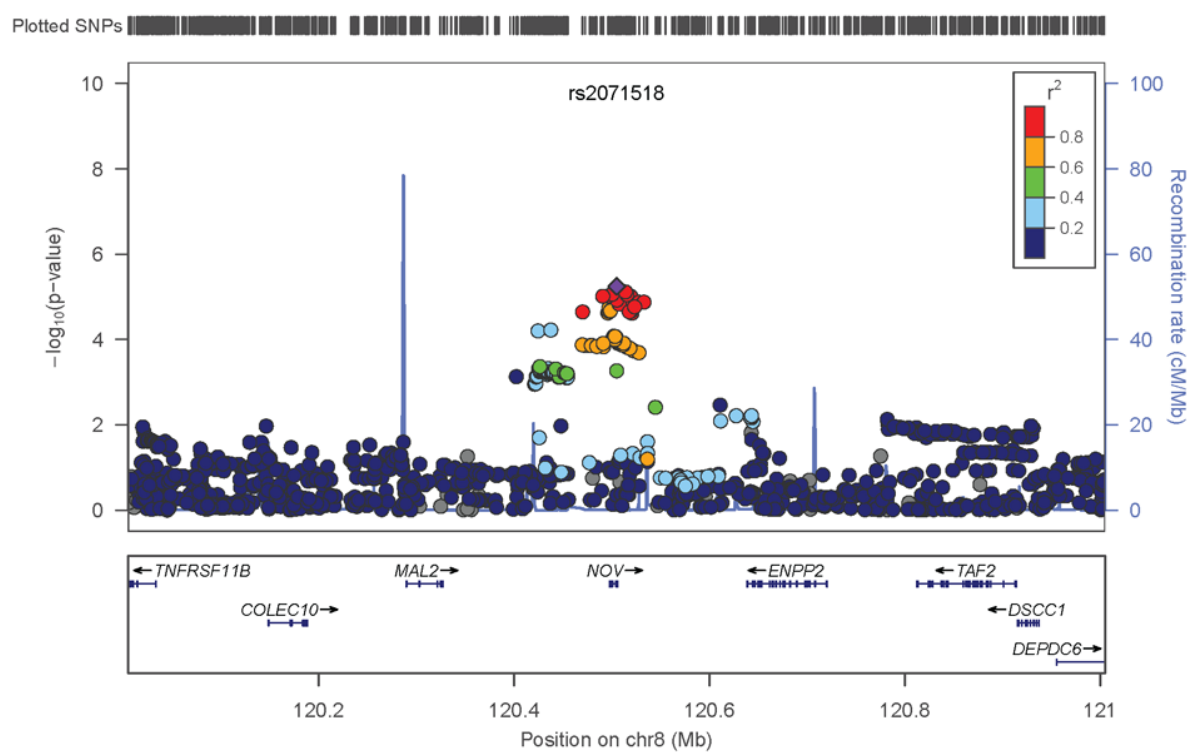

PP rs871606

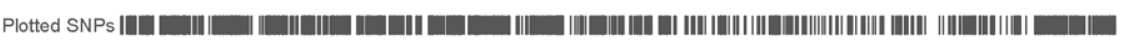

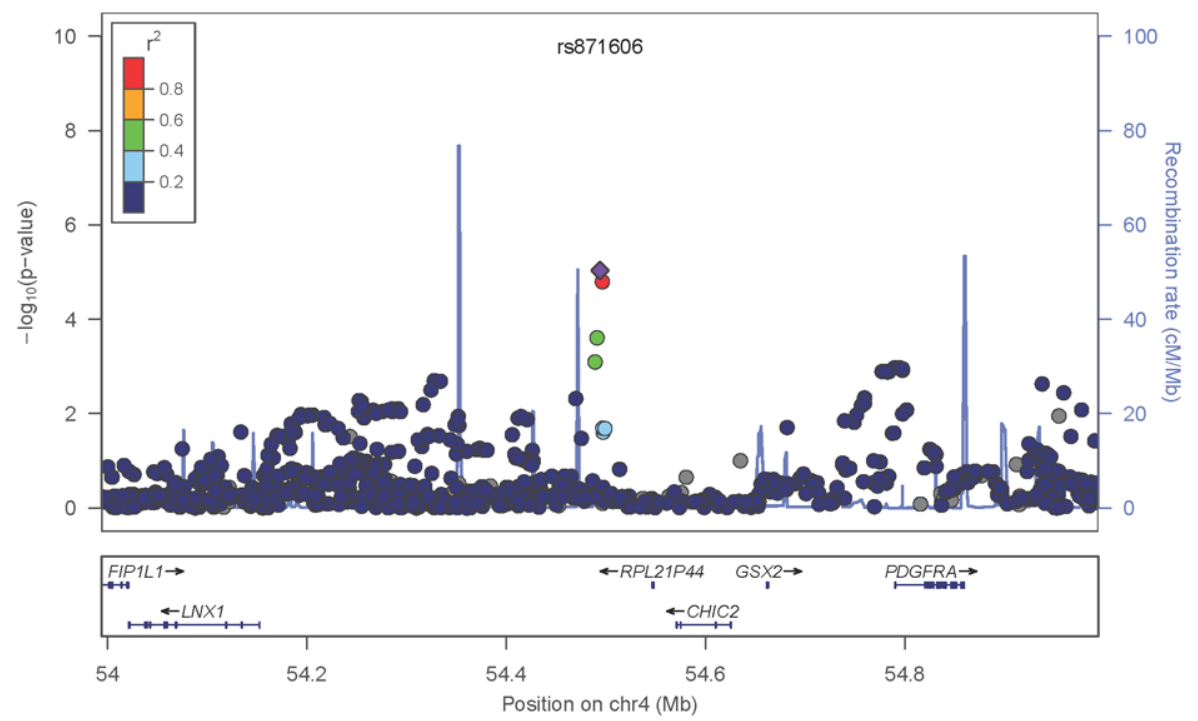

Nat Genet. Author manuscript; available in PMC 2012 September 18. 
MAP rs2782980
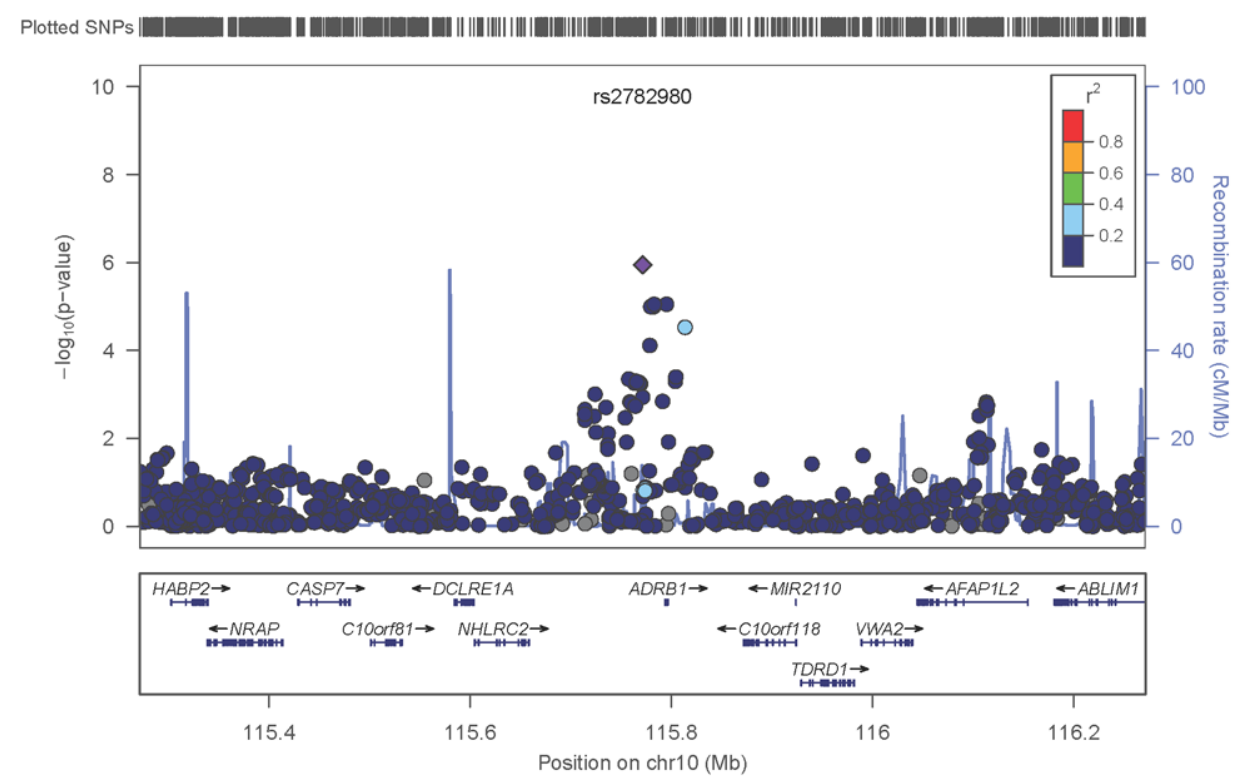

MAP rs319690

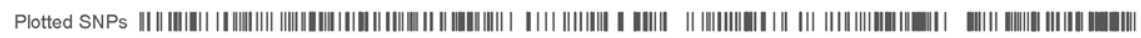

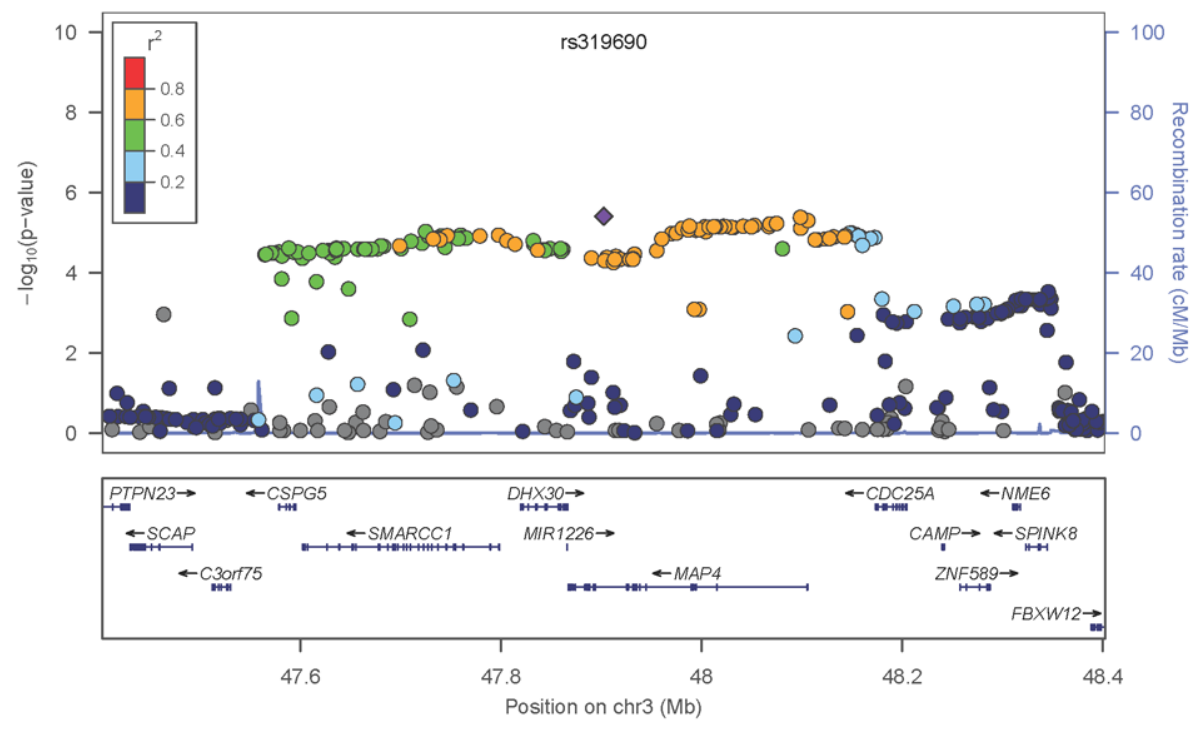

Nat Genet. Author manuscript; available in PMC 2012 September 18. 
PP rs13002573

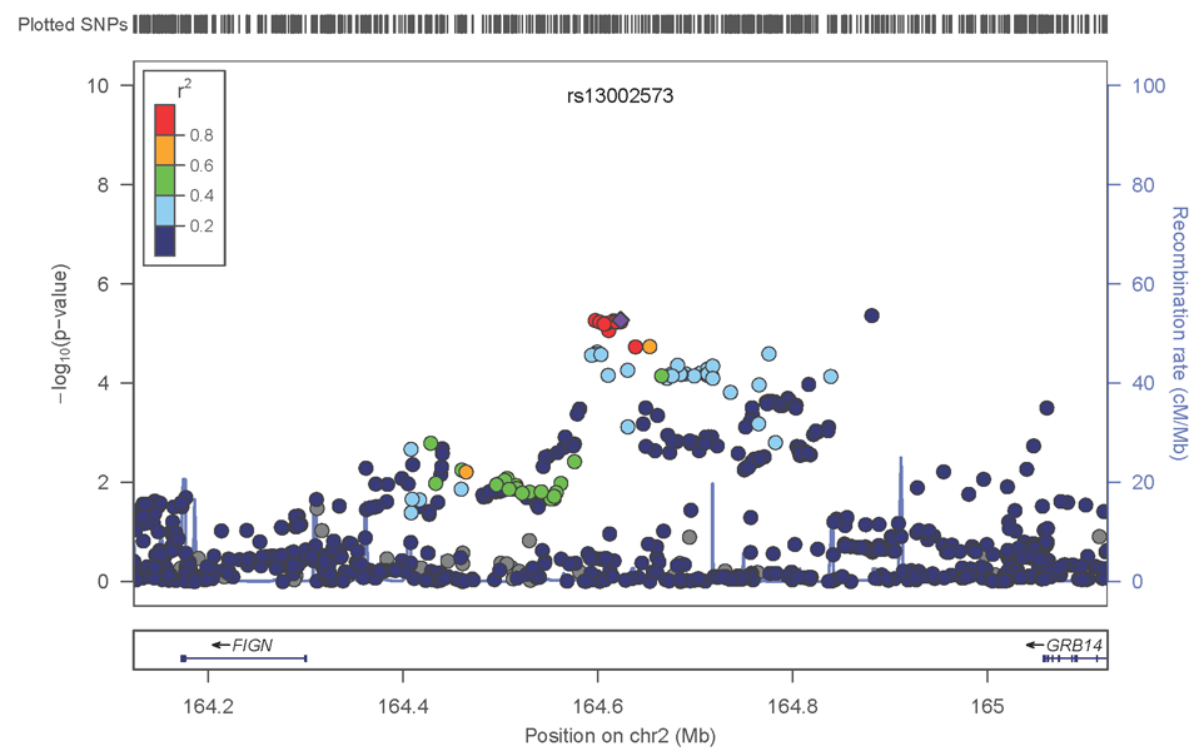

MAP rs 1446468

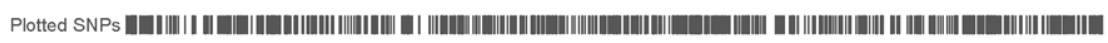

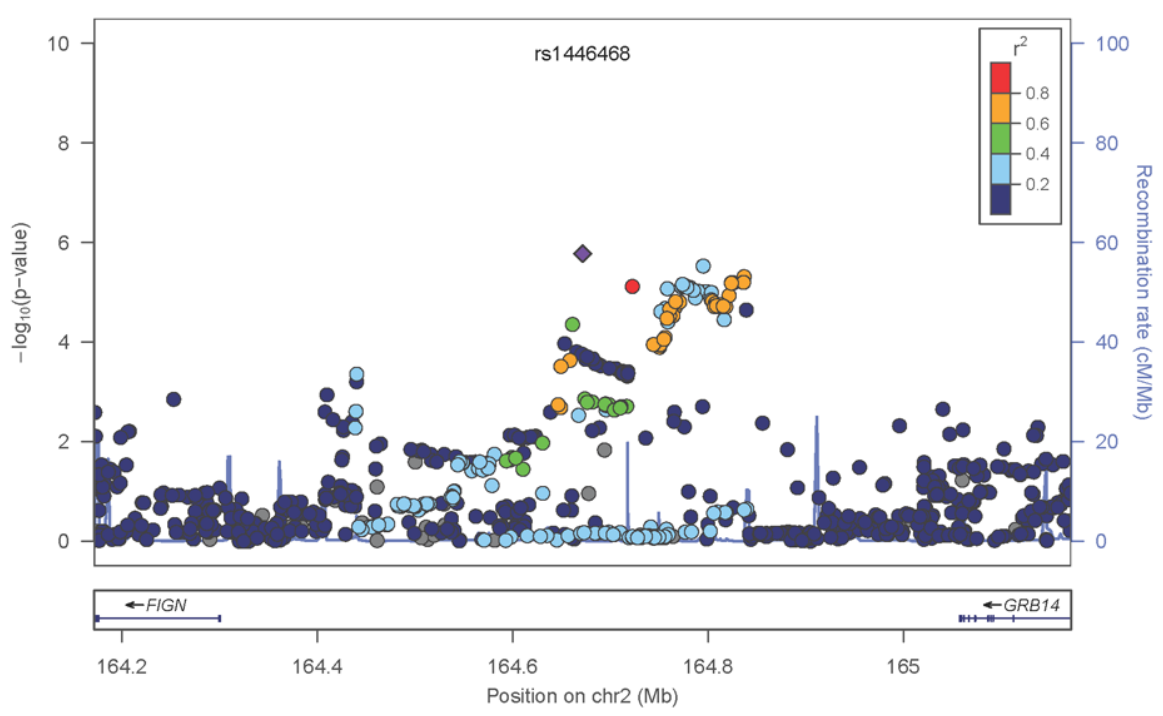

Figure 1.

Regional association plots of the 8 SNPs at 7 loci showing genome-wide significant association $\left(P<5 \times 10^{-8}\right)$ with pulse pressure and/or mean arterial pressure. Statistical significance of each SNP shown on the $-\log _{10}$ scale as a function of chromosome position (NCBI build 36) in the meta-analysis of stage 1 only. The sentinel SNP at each locus is shown in blue; the correlations $\left(\mathrm{r}^{2}\right)$ of each of the surrounding SNPs to the sentinel SNP are shown in the colours indicated in the key. Fine-scale recombination rate is shown in blue. Gene positions are indicated at the bottom. 


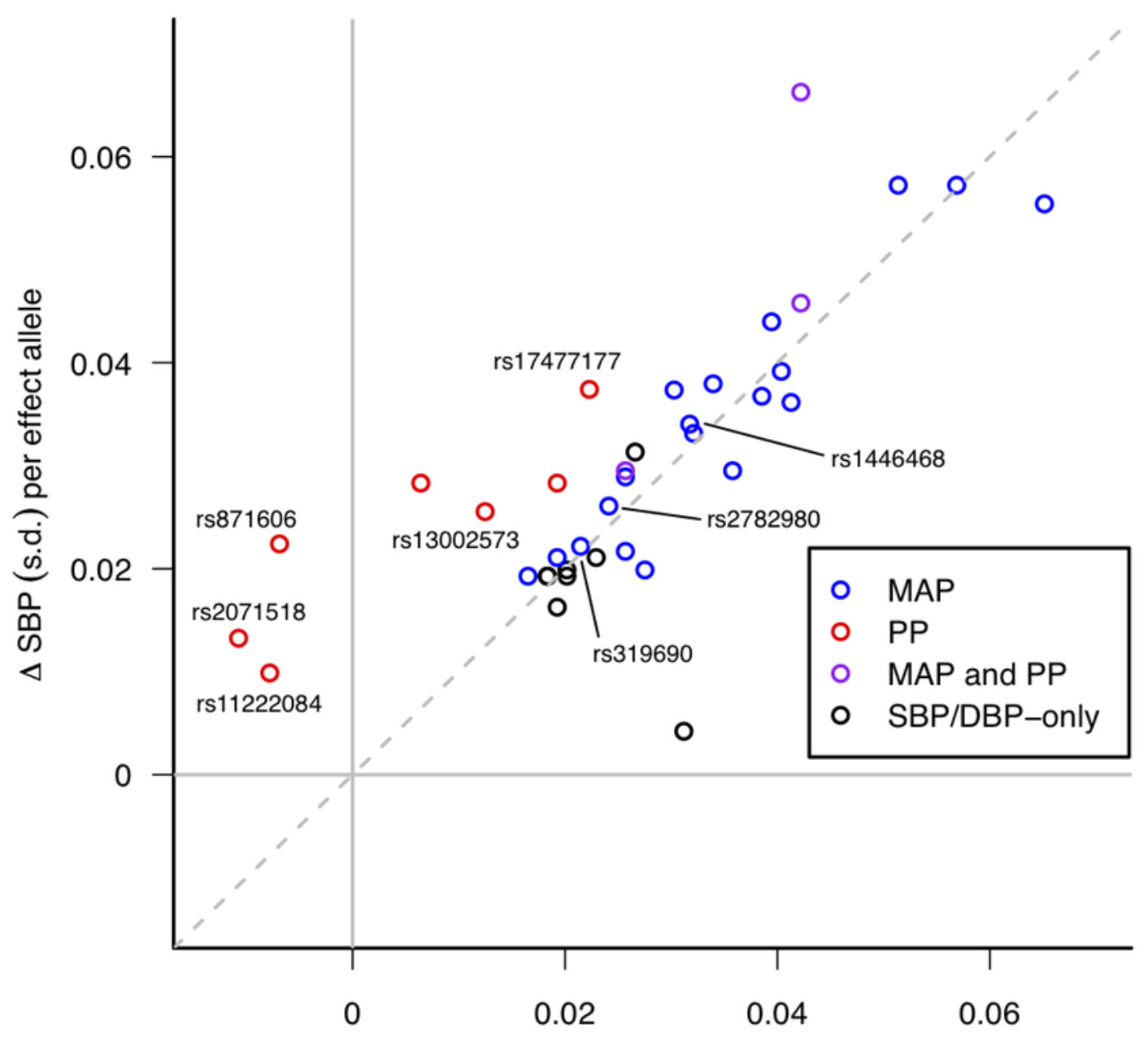

\section{$\Delta$ DBP (s.d.) per effect allele}

Figure 2.

Systolic Blood Pressure (SBP) and Diastolic Blood Pressure (DBP) effect sizes (beta coefficients) for all BP SNPs identified in the present study and Ehret et al. ${ }^{1}$, obtained from follow-up samples only. Beta coefficients are shown as standard deviation (s.d.) differences so that SBP and DBP are measured on comparable scales. Points are colour-coded according to whether they are genome-wide significant $\left(P<5 \times 10^{-8}\right)$ for Pulse Pressure (PP) (red), Mean Arterial Pressure (MAP) (blue) or both PP and MAP (purple) in stages 1 and 2 of the present study, while those that are significant only for SBP and/or DBP from Ehret et al. ${ }^{1}$ are shown in black. The novel SNPs found in the present study are labelled with their rs-numbers. For illustration purposes the effect allele for each SNP is defined such that the direction of the SBP effect is always positive. 
r

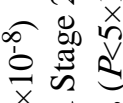
希

$s-\overline{0}$

可

证

की है

言 苍

品 쿵

离 क्षे

कि

똥

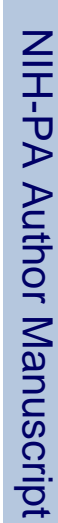

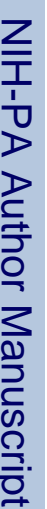

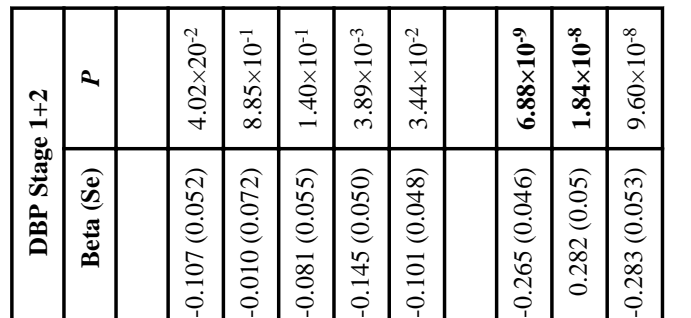

西

क जे

훙

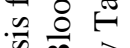

일

㐘

诺

है

ठิ

象

密

을

๙ ज苛

政

至

远专

要

可

它 क

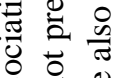

品

票

递要

त

흥

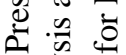

종 흔

过

ซ

$\sum_{0}$

哰

产定

$\Sigma$

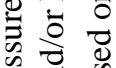

응

品

票声

눙

䒠.

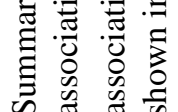

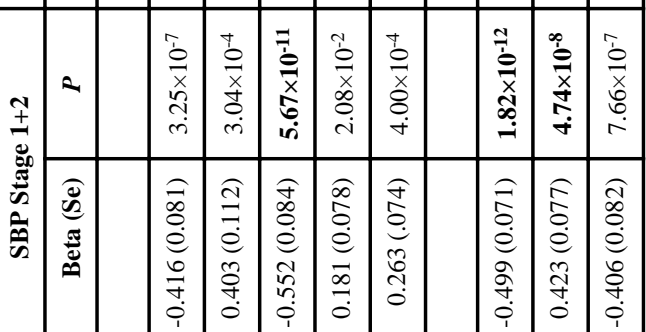

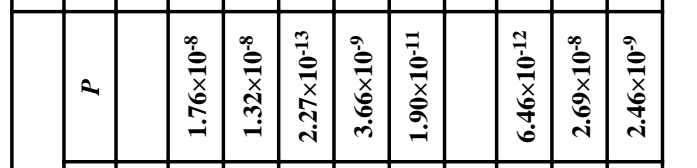

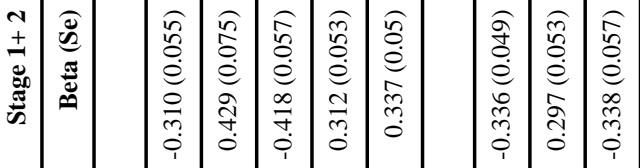

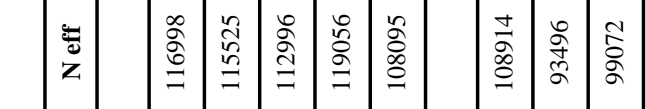

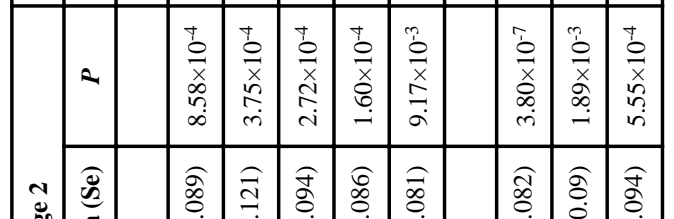

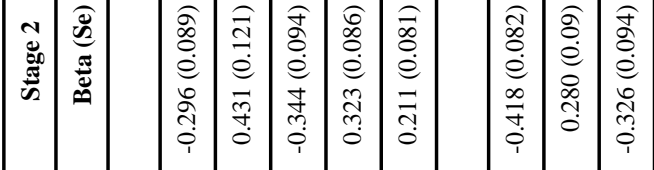

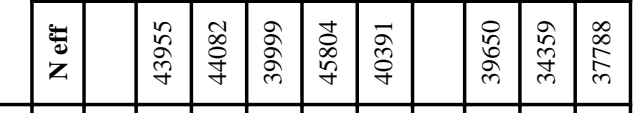

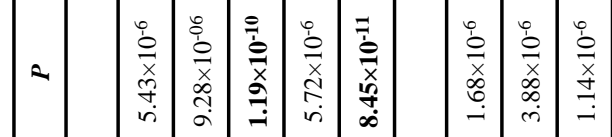

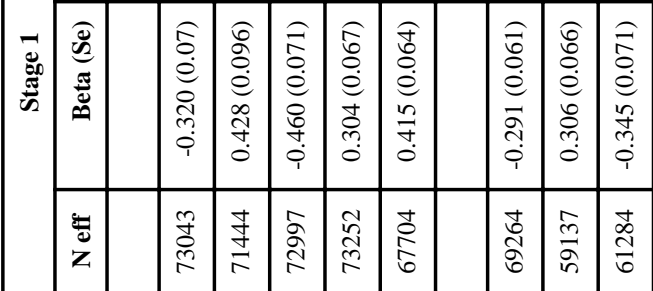

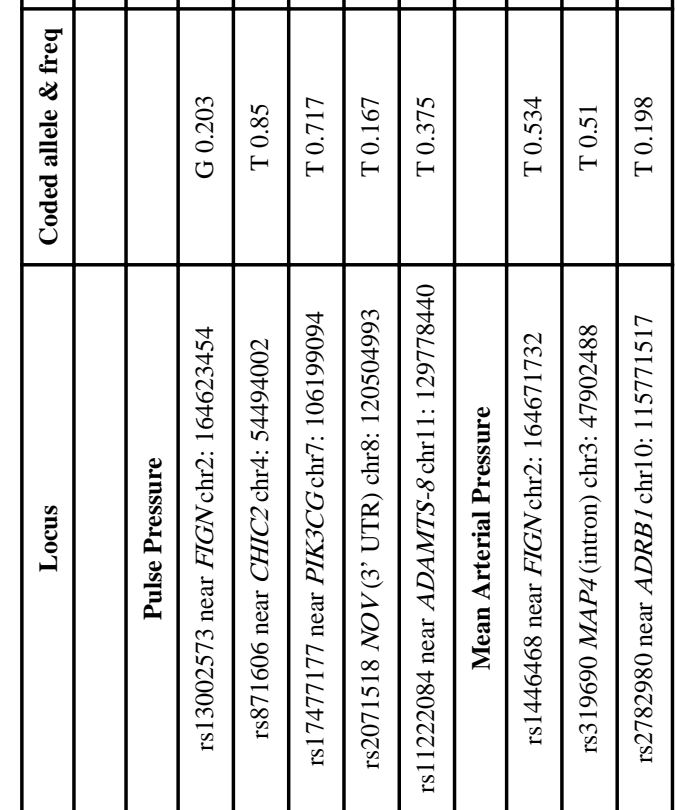

Nat Genet. Author manuscript; available in PMC 2012 September 18. 